Jean Roch Donsimoni, René Glawion, Tobias Hartl, Bodo Plachter, Jens Timmer, Klaus Wälde*, Enzo Weber und Constantin Weiser

\title{
Covid-19 in Deutschland - Erklärung, Prognose und Einfluss gesundheitspolitischer Maßnahmen
}

https://doi.org/10.1515/pwp-2020-0019

Zusammenfassung: Die Autoren erklären den bisherigen Verlauf von Covid-19 in Deutschland durch Regressionsanalysen und epidemiologische Modelle. Sie beschreiben und quantifizieren den Effekt der gesundheitspolitischen Maßnahmen (GPM), die bis zum 19. April in Kraft waren. Sie berechnen den erwarteten Verlauf der Covid-19-Epidemie in Deutschland, wenn es diese Maßnahmen nicht gegeben hätte, und zeigen, dass die GPM einen erheblichen Beitrag zur Reduktion der Infektionszahlen geleistet haben. Die seit 20. April gelockerten GPM sind zwischen den Bundesländern relativ heterogen, was ein Glücksfall für die Wissenschaft ist. Mittels einer Analyse dieser Heterogenität kann aufgedeckt werden, welche Maßnahmen für eine Bekämpfung einer eventuellen zweiten Infektionswelle besonders hilfreich und besonders schädlich sind.

JEL-Klassifikation: I18, E17, C63, C22

Schlüsselwörter: Covid-19, SARS-CoV-2, Corona-Epidemie Deutschland, Prognose, Strukturbruchanalyse, epidemiologische Modelle

Jean Roch Donsimoni, Johannes Gutenberg Universität, Lehrstuhl fü VWL, insb. Makroökonomik, Jakob-Welder-Weg 4, 55118 Mainz, E-Mail: jdonsimo@uni-mainz.de

René Glawion, Universität Hamburg, Fakultät für Wirtschafts- und Sozialwissenschaften, Volkswirtschaftslehre, Von-Melle-Park 5, 20146 Hamburg, E-Mail: Rene.Glawion@uni-hamburg.de Tobias Hartl, Universität Regensburg, Lehrstuhl für Ökonometrie, 93053 Regensburg, E-Mail: tobias1.hartl@ur.de Bodo Plachter, Universitätsmedizin Mainz, Institut für Virologie, Obere Zahlbacher Str. 67, 55131 Mainz,

E-Mail: plachter@uni-mainz.de

Jens Timmer, Universität Freiburg, Physikalisches Institut, CIBSS Centre for Integrative Biological Signaling Studies, Hermann-Herder Str. 3, 79104 Freiburg, E-Mail: jeti@fdm.uni-freiburg.de *Kontaktperson: Klaus Wälde, Johannes Gutenberg Universität, Lehrstuhl für VWL, insb. Makroökonomik, Visiting Research Fellow IZA, Jakob-Welder-Weg 4, 55118 Mainz, E-Mail: waelde@uni-mainz.de Enzo Weber, Institut für Arbeitsmarkt- und Berufsforschung (IAB) der Bundesagentur für Arbeit (BA), Regensburger Straße 104, 90478 Nürnberg, E-Mail: Enzo.Weber@iab.de

Constantin Weiser, Johannes Gutenberg Universität, Fachbereich Rechts- \& Wirtschaftswissenschaften, Quantitative Methodenlehre, Jakob-Welder-Weg 4, 55118 Mainz, E-Mail: c.weiser@uni-mainz.de

\section{Einleitung}

Covid-19 hält die Welt in Atem. Fast genauso atemlos ist die Diskussion über angemessene gesundheitspolitische Maßnahmen (GPM). Eine einfache Möglichkeit zum Bestimmen der Effekte solcher Maßnahmen besteht in einer detaillierten Analyse der vom Robert-Koch-Institut (RKI) und anderen Institutionen täglich veröffentlichten Infektionszahlen. Daraus ergeben sich Hinweise, dass die GPM, die seit 16. März in Kraft sind, erheblich zu einer Reduktion der Ausbreitung von Covid-19 geführt haben.

Will man nicht nur die aktuellen Effekte von Maßnahmen abschätzen, sondern mögliche Verläufe der gesamten Covid-19-Epidemie in Deutschland untersuchen, bieten sich epidemiologische Modelle an. Wir stellen die Grundstruktur solcher Modelle vor und zeigen, was mit ihnen verstanden werden kann. Ebenfalls entwerfen wir ein Modell, das den spezifischen Eigenschaften von Covid-19 Rechnung trägt. Darunter fallen vor allem die sogenannten „stummen Infektionen“, also Infektionen mit CoV-2, die ohne Symptome verlaufen.

Schließlich stellen wir eine aktualisierte Prognose aufbauend auf Daten bis zum 19. April 2020 vor. Wir erörtern den möglichen Verlauf einer "zweiten Welle“ - also eines ab 27. April möglichen neuerlichen Anstiegs der Infektionszahlen. Wir verwenden die aktualisierte Prognose auch, um für aktuelle Beobachtungen bis 23. April sehen zu können, ob die Lockerung von Kontaktsperren ab 20. April bereits Effekte hat. Schließlich präsentieren wir eine ökonometrische Modellstruktur, die uns erlaubt, die seit dem 20. April zwischen Bundesländern sehr heterogenen gesundheitspolitischen Maßnahmen zu evaluieren.

\section{Erklärung über Wachstums- prozesse und Strukturbrüche}

Wir fragen als erstes, wie der bisherige Verlauf von Covid19 in Deutschland über Wachstumsprozesse beschrieben werden kann. Wir verwenden diese Beschreibung zur Bestimmung der Wirksamkeit gesundheitspolitscher Maßnahmen. 
Die Zeitreihe des RKI der mit SARS-CoV-2 gemeldeten Infizierten schien in den Anfangstagen des März geradezu zu explodieren. Die täglichen Zuwachsraten lagen häufig über 50 Prozent, dies war zuletzt am 12. März der Fall (Wälde 2020). Möchte man etwas Hoffnung schöpfen in Zeiten beängstigend hoher Wachstumsraten stellt sich als erstes die Frage, ob diese Raten, wenn schon hoch, dann wenigstens über die Zeit fallen.

Warum sollten Zuwachsraten über die Zeit abnehmen? Dies liegt zum einen in der Natur epidemiologischer Verläufe begründet. Dies sehen wir im Abschnitt 3.3, wo wir epidemiologische Modelle vorstellen, und in Abbildung 5, welche den Verlauf von Covid-19 in Deutschland abbildet. Zuwachsraten von Covid-19-Fällen können aber auch abnehmen, weil Bund und Länder GPM erlassen. Diese Effekte werden wir im Folgenden identifizieren.

\subsection{Hypothesen, Methode und Daten}

Bund und Länder haben verschiedene GPM ergriffen: Seit dem 13. März sind Schulen und Kindergärten geschlossen und große Sportveranstaltungen verboten. Am 22. März einigten sich Bund und Länder darauf, Zusammenkünfte von mehr als zwei Personen zu verbieten und Restaurants sowie Dienstleister wie Friseure zu schließen. Mehrere Bundesländer verhängten zudem noch weiter reichende Sperrmaßnahmen.

Bei einer mittleren Inkubationszeit von 5,2 Tagen (Lauer et al. 2020 und Linton et al. 2020) und einer gewissen Verzögerung zwischen dem Auftreten der Krankheitssymptome, dem Kontaktieren eines Arztes und der Meldung des Falles bei den entsprechenden Behörden (etwa 2-3 Tage) würde man erwarten, dass die Beschlüsse vom 13. März um den 20. März eine erste Wirkung zeigen. Entsprechend sollten Effekte der Beschlüsse vom 22. März um den 29. März sichtbar sein. Finden sich entsprechende Brüche in Zuwachsraten von gemeldet Infizierten?

Eine naheliegende Methode, um die Wachstumsrate von gemeldeten Infizierten zu bestimmen, ist die Regression der Logarithmen der gemeldet Infizierten auf einen linearen Zeittrend. Dabei benutzt man die Tatsache, dass der Logarithmus einer exponentiell wachsenden Zeitreihe die Wachstumsrate dieser Zeitreihe ausgibt. Die genaue Spezifikation unseres Regressionsansatzes ist in Gleichung (1) von Hartl et al. (2020a) dargestellt und dort erläutert. Als Datengrundlage verwenden wir die Werte der Johns Hopkins University (2020). Diese verbindet Daten des deutschen RKI, der Weltgesundheitsorganisation (WHO) und des Europäischen Zentrums für die Prävention und die Kontrolle von Krankheiten (ECDC).

\subsection{Ergebnisse}

Die Beobachtungen sowie die Ergebnisse sind in Abbildung 1 dargestellt.
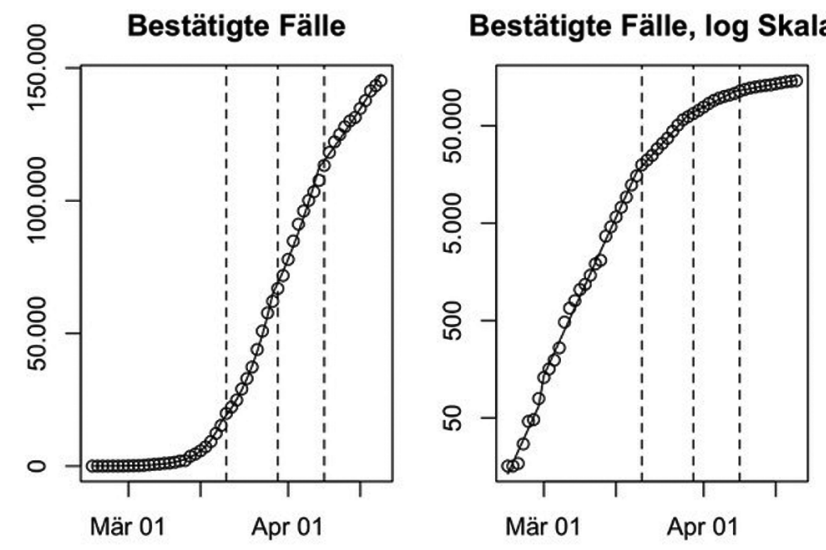

Abbildung 1: Beobachtungen (Kreise) und geschätzte Verläufe (durchgezogene Kurven).

Anmerkung: In der linken Abbildung verwenden wir eine lineare (,normale“) Skala auf der vertikalen Achse, in der rechten Abbildung eine logarithmierte Darstellung. Die Steigung des geschätzten Verlaufs ist damit rechts identisch zur Wachstumsrate. Gestrichelte Linien zeigen Trendbrüche.

Quelle: Eigene Darstellung

Unser log-lineares Trendmodell zeigt Trendbrüche am 20. und 30. März sowie am 8. April. Die drei Trendbrüche weisen für Deutschland auf ein verlangsamtes Wachstum der bestätigten Fälle hin. Alle Trendbrüche sind signifikant auf dem 1-Prozent-Niveau. Die Ergebnisse wurden im Einzelnen wie folgt hergeleitet.

\subsubsection{Messung der Auswirkungen der Richtlinien vom 13. März}

In Hartl et al. (2020a) untersuchten wir die Auswirkungen der am 13. März umgesetzten Richtlinien, d.h. die Schließung von Schulen und Universitäten und die Absage von Massenveranstaltungen. Bei der Suche eines Trendbruchs mittels Maximum Likelihood (Bai 1997 sowie Bai und Perron 1998) fanden wir einen statistisch signifikanten Trendbruch am 20. März, sieben Tage nach der ersten Intervention der Regierungen. Für den Zeitraum vom 23. Februar bis zum 19. März schätzten wir ein tägliches Wachstum von 26,7 Prozent, was bedeutet, dass sich die Fälle alle 2,93 Tage verdoppelten. Ab dem 20. März verringerte sich das durchschnittliche tägliche Wachstum auf 13,8 Prozent, was eine Verdoppelung alle 5,35 Tage bedeutet. 


\subsubsection{Messung der Auswirkungen der Richtlinien vom 22. März}

In Hartl et al. (2020b) beschäftigten wir uns zusätzlich mit den Auswirkungen der Maßnahmen vom 22. März. Wenn die Richtlinien vom 22. März die Verbreitung von Covid-19 beeinflusst haben, dann würde man 7 bis 8 Tage später einen zweiten Rückgang der Wachstumsrate erwarten. Mit den in Hartl et al. (2020a) diskutierten Methoden suchten wir nach einem zweiten Trendbruch.

Wir fanden einen weiteren Strukturbruch am 30. März, der neuerlich das Wachstum der Covid-19-Fälle reduziert. So kann acht Tage nach der Implementierung der Maßnahmen eine Verringerung der Wachstumsrate von Covid19 festgestellt werden. Unsere Ergebnisse zeigten, dass das durchschnittliche Fallwachstum vor dem 19. März 26,7 Prozent betrug. Entsprechend verdoppelten sich hier die Fallzahlen alle 2,93 Tage. Zwischen 20. März und 29. März fanden wir ein mittleres Wachstum von 13,6 Prozent, was Verdopplungen alle 5,42 Tage bedeutet. Ab dem 30. März lag die durchschnittliche Wachstumsrate bei 5,8 Prozent, und die Fälle verdoppelten sich alle 12,2 Tage.

\subsubsection{Berücksichtigung der Daten bis zum 19. April}

Unser neuer Beitrag in diesem Papier besteht in der Suche nach einem weiteren Trendbruch. Unter Berücksichtigung der Daten bis zum 19. April finden wir diesen mit den gleichen Methoden wie oben tatsächlich am 8. April. Für den Wachstumspfad der Covid-19-Fallzahlen bedeutet das folgendes: Zwischen dem 23. Februar und dem 19. März betrug die mittlere tägliche Wachstumsrate der Covid-19Fälle 26,7 Prozent, was bedeutet, dass sich die Fälle alle 2,93 Tage verdoppelt haben. Vom 20. März bis zum 29. März betrug die durchschnittliche Wachstumsrate 13,6 Prozent, was bedeutet, dass sich die Fälle alle 5,45 Tage verdoppelten. Von 30. März bis 7. April schätzen wir eine durchschnittliche Wachstumsrate von 6,1 Prozent, so dass sich die Fälle alle 11,68 Tage verdoppelten. Ab dem 8. April liegt die geschätzte durchschnittliche Wachstumsrate bei 2,3 Prozent, was eine Verdopplung der Fallzahlen alle 31,13 Tage nach sich zieht. Geringe Abweichungen zu den Ergebnissen aus Hartl et al. (2020b) sind dadurch zu erklären, dass wir mittlerweile über eine längere Zeitreihe an Beobachtungen verfügen.

\section{Prognosen über mathematische Modelle}

Die bisherige Analyse hat gezeigt, dass GPM dazu beigetragen haben, die Ausbreitung von Covid-19 in Deutschland erheblich zu reduzieren. Möchte man nicht nur die aktuelle Lage einschätzen, sondern ein Gefühl dafür entwickeln, welcher Gesamtverlauf für Covid-19 in Deutschland zu erwarten ist, bieten sich mathematische Modelle an.

Mathematische Modelle beinhalten Parameter, welche für informative Prognosen realistische Werte zugeordnet bekommen müssen. Einige Parameterwerte folgen direkt aus aktuell beobachteten Infektionszahlen, andere Parameterwerte werden für Covid-19 vermutlich erst in einigen Jahren vorliegen. Zum Abschätzen der Plausibilität möglicher Werte werfen wir zunächst einen Blick in die Geschichte größerer Pandemien.

\subsection{Historische Einordnung von Covid-19}

Die durch das neuartige Coronavirus SARS-CoV2 verursachte Erkrankung (Covid-19) nahm nach aktuellem Verständnis ihren Ausgangspunkt 2019 in der Stadt Wuhan in der Provinz Hubei, China. ${ }^{1}$ Die schwere Verlaufsform der Erkrankung ist durch akutes Lungenversagen im Vollbild eines „Severe Accute Respiratory Syndrome“ (SARS) gekennzeichnet.

\subsubsection{Epidemietypen und historische Beispiele}

Grundsätzlich kann zwischen viralen und nicht-viralen Pandemien unterschieden werden. Beispiele für virale Pandemien sind die Grippe, AIDS oder eben auch Covid19. Durch Bakterien ausgelöste Pandemien beinhalten unter anderem die Pest und Fleckfieber. Wir beschränken uns im Folgenden auf Pandemien im 20. Jahrhundert, da unterschiedliche gesellschaftliche und medizinische Rahmenbedingungen früherer Jahrhunderte Rückschlüsse auf die aktuelle Pandemie weitgehend ausschließen. ${ }^{2}$ Einen Überblick ausgewählter Pandemien des 20. Jahrhunderts bietet folgende Tabelle.

1 Wilder-Smith et al. (2020) beschreiben Unterschiede und Gemeinsamkeiten zwischen der SARS-2003-Epidemie und Covid-19. 2 Alfani (2020) betrachtet auch weiter zurückliegende Pandemien. 
Tabelle 1: Statistische Kenngrößen für Pandemien aus dem 20. Jahrhundert

\begin{tabular}{llll}
\hline Pandemie & $\begin{array}{l}\text { Asymptomati- } \\
\text { sche Fälle }\end{array}$ & $\begin{array}{l}\text { Infektionsraten } \\
\text { (in aller Welt) }\end{array}$ & $\begin{array}{l}\text { Sterberaten pro } \\
100.000 \\
\text { Personen }\end{array}$ \\
\hline $\begin{array}{l}\text { Spanische } \\
\text { Grippe } 1918\end{array}$ & $<6 \%$ & $28 \%$ & 218 \\
\hline $\begin{array}{l}\text { Asiatische } \\
\text { Grippe 1957 }\end{array}$ & $\approx 8 \%$ & $24 \%^{1}$ & 22 \\
\hline $\begin{array}{l}\text { Hongkong } \\
\text { Grippe 1968 }\end{array}$ & - & $13 \%^{2}$ & 13,9 \\
\hline $\begin{array}{l}\text { Saisonale } \\
\text { Grippe }\end{array}$ & $25-75 \%$ & $9 \%$ & $4,0-8,8$ \\
\hline
\end{tabular}

Anmerkungen: ${ }^{1}$ USA, ${ }^{2}$ Hongkong. Infektionsraten stehen für die Infizierten, die Symptome zeigen.

Quelle: Die Sterblichkeitsraten für die saisonale Grippe sind aus Juliano et al. (2018), für die Spanische, Asiatische, und Hongkong Grippen aus Glezen (1996).

\subsubsection{Symptome, Infektionsraten und Sterberaten}

Ein Kennzeichen von Covid-19 ist ihr teilweise asymptomatischer Verlauf, also eine Infektion ohne erkennbare Krankheitssymptome. Auch wenn der aktuelle Forschungsstand keine sicheren Aussagen zulässt, wird vermutet, dass zu jedem erfassten Infizierten zwischen 5 und 10 nicht erfasste Infizierte gehören. Das würde bedeuten, dass bis zu 90 Prozent aller CoV-2-Infektionen asymptomatisch verlaufen. Historisch betrachtet erscheint dies im Vergleich zu den großen Pandemien ungewöhnlich. Fraser et al. (2011) schätzen für die Grippewelle von 1917/18 (,Spanische Grippe“), dass nur etwa 6 Prozent der Infektionen asymptomatisch verliefen. Jackson (2009) schätzt diesen Anteil für die Asiatische Grippe von 1957 auf etwa 8 Prozent. Bei den jährlichen Grippewellen wird der Anteil jedoch mit 25-75 Prozent viel höher geschätzt (Fragaszy et al. 2017).

Fragt man nach dem Anteil der Infizierten am Ende einer Epidemie ${ }^{3}$, so gibt die mittlere Spalte in der Tabelle Auskunft. Die Grippe von 1918 infizierte in aller Welt etwa 28 Prozent der Bevölkerung (Glezen 1996, Taubenberger und Morens 2006 sowie Taubenberger 2006). Auf eine

3 Eine Pandemie endet, wenn die globale Ausbreitung eingedämmt ist und die Zirkulation des Virus nurmehr lokal nachzuweisen ist oder ganz verschwindet. Nach dieser Definition läuft zum Beispiel die HIV-Pandemie immer noch. Das gleiche gilt für die „Schweinegrippe“. Das Virus verursacht, als einer von vier Stämmen, jeden Winter neuerlich Epidemien. Bezüglich SARS-CoV-2 hofft man eines Tages auf eine nurmehr lokale Zirkulation. ähnliche Größenordnung kommt man mit 24 Prozent (der amerikanischen Bevölkerung) bei der Asiatischen Grippe von 1957 (Glezen 1996). Die Hongkong-Grippe infizierte innerhalb weniger Monate mindestens 13 Prozent der Bevölkerung Hongkongs (Starling 2006). ${ }^{4}$ Im Gegensatz zu diesen geringen Infektionsraten erfasst die gewöhnliche Grippe etwa 20 Prozent der Bevölkerung der Vereinigten Staaten bzw. fast 9 Prozent der Weltbevölkerung (Clayville 2011). Für Deutschland liegen diese Zahlen zwischen 1 und 2 Prozent in den Jahren 2012 bis 2014 und bei bis zu 4,5 Prozent 2018/2019 (Scholz et al. 2019 und Robert-KochInstitut 2019). Symptome sind jedoch selten schwer oder gar lebensbedrohlich.

Die Sterberaten in obiger Tabelle zeichnen ein klares Bild der Gefährlichkeit einzelner Epidemien. Die Spanische Grippe war 25 bis 50-mal tödlicher als die gewöhnliche Grippe und fast 10-mal tödlicher als die Asiatische Grippe von 1957.

\subsubsection{Wann enden Epidemien?}

Eine für die aktuelle Situation nicht ganz unerhebliche Frage ist die nach dem Ende dieser historischen Pandemien. Die Asiatische Grippe und die Hongkong-Grippe endeten mit einer Kombination aus der Entwicklung von Impfstoffen, nicht-pharmazeutischen Interventionen (NPI) und einer sich langsam entwickelnden Immunität in der Bevölkerung (Kilbourne 2006 sowie Saunders-Hastings und Krewski 2016). Da die Grippe von 1918 vor der Entdeckung ihrer viralen Ursache ausbrach, blieben den Behörden nur NPI wie (man mag sich an heute erinnert fühlen) Quarantäne, verbesserte Hygienestandards und das Schließen öffentlicher Gebäude (Saunders-Hastings und Krewski 2016). Dies führte zu einem Ende der Pandemie, das pandemische Virus hingegen verursachte weiterhin jährliche Epidemien.

\subsection{Epidemiologische Modelle}

Schauen wir uns nun zunächst ein klassisches epidemiologisches Modell an und fragen uns, was wir damit prinzipiell verstehen können.

4 Viel kleiner sind im Vergleich dazu die Infektionsanteile der NichtPandemie SARS 2002. Sie lagen bei nur 26 von 100.000 Personen (0,026 Prozent) bzw. 6 von 100.000 Personen (0,006 Prozent) in Hongkong bzw. Singapur (World Health Organization 2004). 


\subsubsection{Modelltypen}

In der Epidemiologie gibt es eine lange Tradition mathematischer Modelle. Diese beschreiben die Ausbreitung eines Erregers oder einer Krankheit in einer Population. Mathematische Modelle werden aus zwei Gründen entwickelt. Sie können einerseits allgemeine qualitative Einsichten liefern, andererseits erlauben sie, konkrete quantitative Vorhersagen zu treffen.

Modelle werden in der Literatur unterschiedlich klassifiziert (siehe Adam 2020 für einen nicht-technischen Überblick). Wichtige Modelltypen sind agentenbasierte Modelle, stochastische Simulationsmodelle und deterministische Modelle (siehe zum Beispiel Allen 2008). Die Unterscheidung zwischen stochastischen und deterministischen Modellen erscheint teilweise irreführend. Schaut man sich die deterministischen Modelle genauer an (Allen 2008 sowie Donsimoni et al. 2020a), erkennt man die dahinterstehenden stochastischen Konzepte (Markovketten in kontinuierlicher Zeit). Diese werden jedoch durch Erwartungswerte beschrieben, deren Verlauf in der Tat deterministisch ist.

\subsubsection{Die „Susceptible-Infectious-Removed“-Modelle (SIR)}

Eine große Klasse von „deterministischen“ Modellen sind die sogenannten SIR-Modelle (siehe Hethcote 2000). Das erste und einfachste Modell zur Beschreibung des zeitlichen Verlaufs einer Epidemie haben Kermack und McKendrick 1927 aufgestellt. Es geht von drei Populationen aus: von den Suszeptiblen $(S)$, also Gesunden, die sich anstecken können; den Infektiösen (I), die Suszeptible anstecken können; und von einer im Englischen als „Removed“ $(R)$ bezeichneten Gruppe von Menschen, die an der Infektion verstorben oder aber genesen und immun sind. Graphisch lässt sich dies als $S(t) \rightarrow I(t) \rightarrow R(t)$ darstellen, wobei $S(t)$ für die Anzahl der Gesunden zum Zeitpunkt $t$ steht, $I(t)$ für die Anzahl der Infizierten und $R(t)$ für die zum Zeitpunkt $t$ Verstorbenen bzw. Genesenen.

Wenn man die Populationsdynamik genau verstehen möchte, drückt man die zeitliche Änderung von Populationsgrößen durch Differentialgleichungen aus,

$\dot{S}(t)=-r I(t) S(t), \dot{I}(t)=r I(t) S(t)-a I(t), \dot{R}(t)=a I(t)$.

Dabei beschreibt beispielsweise $\dot{S}(t)$ die Ableitung von $S(t)$ nach der Zeit, drückt also die Änderung von $S(t)$ aus (und analog für $I(t)$ und $R(t)$ ). Zentral zum Verständnis von Epidemien ist die Geschwindigkeit, mit der Individuen erkranken und genesen, also von $S$ nach $I$ und dann nach $R$,wandern“. Diese Übergangsgeschwindigkeiten, genau gesagt diese Übergangsraten, sind in obigen Gleichungen sichtbar. Die Infektionsrate ist $r I(t)$, wobei $r$ die Infektionsratenkonstante ist. Die Infektionsrate ist die Wachstumsrate, mit der der Bestand an Gesunden $S(t)$ fällt. Diese Rate beschreibt auch die Neuerkrankungen, also den Zufluss in den Zustand $I(t)$. Die Rate, mit der infizierte Individuen genesen bzw. versterben, ist mit $a$ bezeichnet. ${ }^{5}$

\subsubsection{Grundlegende Konzepte}

Mithilfe dieser Modelle können die vielen Größen, über die in der Öffentlichkeit diskutiert wird, genau beschrieben und damit verstanden werden. Fragen wir als erstes: Wann wird eine Infektion zur Epidemie? Dies geschieht, wenn die rechte Seite der Gleichung $\dot{I}(t)$ für $t=0$ größer als 0 ist, da dann die Anzahl der anfänglich Infizierten wächst. Ist sie kleiner als 0, stirbt die Infektion aus. In diesem Zusammenhang ist es hilfreich, die sogenannte Reproduktionszahl

$\operatorname{Rep}(t)=\frac{r S(t)}{a}$

einzuführen. ${ }^{6}$ Sie beschreibt, wie viele Suszeptible ein Infektiöser im Mittel zu einem Zeitpunkt $t$ ansteckt. Da die Anzahl der Gesunden $S(t)$ über die Zeit abnimmt, sinkt auch die Reproduktionszahl. Die Bedeutung der Reproduktionszahl wird klar, wenn man unter Verwendung von $\operatorname{Rep}(t)$ die Gleichung für $\dot{I}(t)$ in der Form

$\dot{I}(t)=(r S(t)-a) I(t)=(\operatorname{Rep}(t)-1) a I(t)$

schreibt: Die Anzahl der Infizierten nimmt zu; eine Infektion wird zur Epidemie, wenn die Reproduktionszahl größer 1 ist, ansonsten nimmt sie ab.

Wie verhält sich die Epidemie zu Beginn? Zu Beginn der Epidemie gibt es nur wenige Infektiöse. Dies hat zur Folge, dass nur wenige Suszeptible infiziert werden, womit

5 Aus der Volkswirtschaftslehre sind die „Search \& matching“-Modelle nach Diamond (1982), Mortensen (1982) und Pissarides (1985) bekannt. Einfache Versionen davon werden manchmal als „Badewannenmodell“ bezeichnet. In diesen Modellen geht es um den $\mathrm{Zu}$ fluss in die Arbeitslosigkeit und um den Abfluss in die Beschäftigung. In den SIR-Modellen geht es um den Zufluss in die Infektion und um den Abfluss in Genesung oder Tod.

6 Die Reproduktionszahl wird in der gesamten epidemiologischen Literatur mit $R(t)$ bezeichnet. Da $R(t)$ jedoch auch die Genesenen bzw. Verstorbenen („removed“) bezeichnet, verwenden wir hier $\operatorname{Rep}(t)$ für die Reproduktionszahl. 
$S$ im Wesentlichen konstant ist. Die Gleichung für den Anstieg der Infizierten kann dann als

$\dot{I}(t)=(\operatorname{Rep}(0)-1) a I(t)$

geschrieben werden. Hierbei ist nun $\operatorname{Rep}(0)=r S(0) / a$ die Reproduktionszahl zum Zeitpunkt $t=0$ oder auch die Basisreproduktionszahl. Hier erkennt man den nun bekannten Zusammenhang: Wenn die Basisreproduktionszahl größer 1 ist, dann kommt es zu einem exponentiellen Wachstum, bei einem Wert kleiner 1 zu einem Aussterben.

\subsubsection{Antworten des Modells}

Warum endet eine Epidemie? Schon die obigen Gleichungen zeigen, dass die Anzahl der Infizierten sinkt, wenn es entweder keine Gesunden mehr gibt, $S(t)=0$, oder wenn die Anzahl der Infizierten $I(t)$ gleich Null ist. Das bedeutet, dass wir mitnichten darauf warten müssen, bis alle mit CoV-2 infiziert sind, sondern dass es schon ausreicht, wenn es keine Infizierten mehr gibt, also $I(t)=0$.

Wie soll man eine Epidemie bekämpfen? Da das Ziel ist, $\operatorname{Rep}(t)$ unter 1 zu drücken, muss man entweder $r$ reduzieren oder die Anzahl der Suszeptiblen. Bei letzterem setzen Impfungen an, die uns aber leider für Covid-19 noch nicht zu Verfügung stehen. Allerdings sorgt die Epidemie selbst für eine Reduktion der Suszeptiblen, da diejenigen, die die Infektion überlebt haben, immun sind.

Wenn man das Ziel verfolgt, Neuinfektionen zu vermeiden, dann kann dies durch Quarantänemaßnahmen erfolgen. Könnte man alle Infizierten identifizieren (was aktuell nicht möglich ist), dann fänden keine Neuinfektionen statt; die Infektionsrate $r I(t)$ wäre Null. Die Anzahl der Gesunden $S(t)$ bliebe konstant, die Anzahl der (noch) Infizierten sänke.

Was soll man konkret tun? Die sicherste Methode, eine Epidemie $\mathrm{zu}$ beenden, ist die Immunisierung von Suszeptiblen, die dafür sorgt, dass ein Infizierter im Mittel nur noch einen, besser weniger als einen Suszeptiblen ansteckt. Dafür müssen von den $\operatorname{Rep}(t)$ Suszeptiblen, die ein Infizierter ohne jede Immunisierung anstecken würde, $\operatorname{Rep}(t)-1$ immun sein. In Gleichungen ergibt sich damit der Anteil der Bevölkerung, der immun sein muss, als

7 Daraus folgt auch, dass eine lokale Infektion eine umso geringere Chance hat, zur Epidemie zu werden, je schneller sie tödlich verläuft, oder - im positiven Fall - je schneller die Infizierten gesunden und sich Immunität einstellt. Daher ist das Ebola-Virus global gesehen weniger gefährlich als das AIDS auslösende HI-Virus, da mit letzterem Infizierte jahrelang symptomfrei, aber infektiös sein können.
$(\operatorname{Rep}(t)-1) / \operatorname{Rep}(t)$

Was sagt uns dies für Covid-19 in Deutschland? Für die Corona-Epidemie wurde $\operatorname{Rep}(0)$ auf etwa 2,5 geschätzt (Zhang 2020). Dies bedeutet, dass sich die sogenannte Herdenimmunität einstellt, wenn mindestens 60 Prozent der Bevölkerung immun sind. Dafür müssten sie allerdings vorher infiziert und damit dem Risiko zu sterben ausgesetzt sein und das Gesundheitssystem liefe Gefahr, überlastet zu werden. Für Deutschland bedeutet dies, dass sich etwa 50 Millionen Menschen infizieren müssten. Bei einer angenommenen Sterberate von einem Prozent würde dies 500.000 Tote und einen Zusammenbruch des Gesundheitssystems bedeuten.

Die zweite Möglichkeit, $\operatorname{Rep}(t)$ unter die $1 \mathrm{zu}$ drücken - die Reduktion des Infektionsparameters $r$ - besteht in der sogenannten sozialen Distanzierung. Dies ermöglicht eine Beendigung der aktuellen Epidemie, birgt aber die Gefahr ihrer Wiederkehr, sobald die Vorsichtsmaßnahmen wieder aufgehoben werden, da sich bis dahin keine Herdenimmunität eingestellt hat. Die Hoffnung wäre, dass die Zahl der Infizierten bei einem neuen Ausbruch der Epidemie so gering ist und die Bevölkerung hinreichend sensibilisiert, dass sich Infektionsketten gut rekonstruieren lassen und gezielt Menschen in Quarantäne genommen werden könnten.

\subsubsection{Erweiterung des Grundmodells}

Basierend auf dem SIR-Modell sind Erweiterungen vorgenommen worden, die zum Beispiel berücksichtigen, dass ein Individuum nach seiner Infektion nicht sofort selbst infektiös ist (SEIR-Modelle) oder dass Genesene nicht immun sind (SIS-Modelle). Alle diese Modelle (siehe Hethcote 2000) stellen deterministische Betrachtungen an und gelten somit nur für große Fallzahlen.

Für kleine Fallzahlen - die Grenze liegt in der Größenordnung von 100 - muss man den Zufallsmomenten des Ansteckungsprozesses Rechnung tragen. Dies führt zu stochastischen Differentialgleichungen. Sogenannte Agenten-basierte Modelle gehen noch einen Schritt weiter und simulieren - ebenfalls häufig stochastisch - einzelne Menschen und beispielsweise ihr Bewegungsverhalten. Eine weitere Verallgemeinerung dieser Modelle besteht darin, die Menschen in unterschiedliche Gruppen einzuteilen, sie zu Beispiel nach Geschlecht, Alter oder Vorerkrankungen zu unterscheiden, und dann unterschiedliche Modellparameter für unterschiedlichen Gruppen zu wählen. 


\subsubsection{Der Forschungsrand}

In einem viel beachteten Bericht einer Arbeitsgruppe vom Imperial College London (Ferguson 2020) wurde die Situation in Großbritannien modelliert. Hierzu nutzen die Autoren ein Alters-strukturiertes Agenten-basiertes Modell, das naturgemäß viele Parameter und Annahmen enthält, die nicht genau bekannt sind. Sie untersuchen die Auswirkungen von fünf verschiedenen Varianten der sozialen Distanzierung und ihrer Kombinationen. Sie schlagen eine On-off-Strategie vor, bei der die Maßnahmen vorgenommen werden, wenn die Zahl der Infizierten einen bestimmten Wert überschreitet und gelockert werden, wenn diese einen bestimmten Wert unterschreitet. Die Autoren kommen zu dem wenig ermutigenden Schluss, dass diese Strategie durchgehalten werden muss, bis ein Impfstoff zur Verfügung steht.

\subsection{Das aktuelle Prognosemodell}

Für das Verständnis von Covid-19 muss das oben vorgestellte SIR-Modell erweitert werden. Der Hauptgrund besteht darin, dass keine Unterscheidung zwischen beobachteten und nicht-beobachteten Infektionen getroffen wird. Diese „stummen Infektionen“ sind für ein Verständnis von Covid-19 jedoch entscheidend.

\subsubsection{Das Modell}

Donsimoni et al. (2020a, b) haben ein epidemiologisches Modell für Covid-19 entwickelt, das Infektionen ohne Symptome berücksichtigt. Es sind also sowohl gemeldete SARS-Cov2-Infektionen abgebildet als auch stumme Infektionen. ${ }^{8}$ Dieses Modell wurde für Deutschland mit Daten ab 24. Februar 2020 kalibriert und zur Prognose verwendet. Die Prognose erfolgte mit Hilfe der Daten des RKI bis zum 21. März und stellt damit einen hypothetischen Verlauf der Covid-19-Epidemie in Deutschland unter Abwesenheit jeglicher behördlicher Interventionen vor. Donsimoni et al. (2020c) haben die Prognose aktualisiert und den Effekt der Kontaktsperren ab 15. März 2020 betrachtet. Das Modell ist grafisch in Abbildung 2 dargestellt.

Die Bevölkerung der Bundesrepublik befindet sich in diesem Modell am 24. Februar 2020 am Beginn der Epi-

8 Die Anzahl der gemeldeten Infizierten hängt auch von der Anzahl der durchgeführten Tests ab. Diesen Einfluss haben wir nicht explizit berücksichtigt. Nach unserem besten Wissen wurde dieser Aspekt auch in der Literatur bisher noch nicht untersucht. demie. Damals waren 16 Personen beim RKI als infiziert gemeldet. Der Rest der 83,1 Millionen befand sich entweder im Zustand „gesund“ oder im Zustand ,infiziert, nicht gemeldet“. Dieser ist in der Abbildung gestrichelt dargestellt, da er im erwähnten Modell nicht als expliziter Zustand aufgeführt ist. Als Erweiterung gegenüber vielen SIR-Modellen verwenden Donsimoni et al. (2020a, b, c) jedoch explizit einen Übergang von $S$ nach $R$, der nicht über ,infiziert und gemeldet“ geht, da unter anderem viele Infektionen mit CoV-2 asymptomatisch verlaufen und damit unentdeckt bleiben. Eine gemeldete Infektion kann in zwei Ausprägungen von $R$ („removed“) enden, verstorben oder genesen.

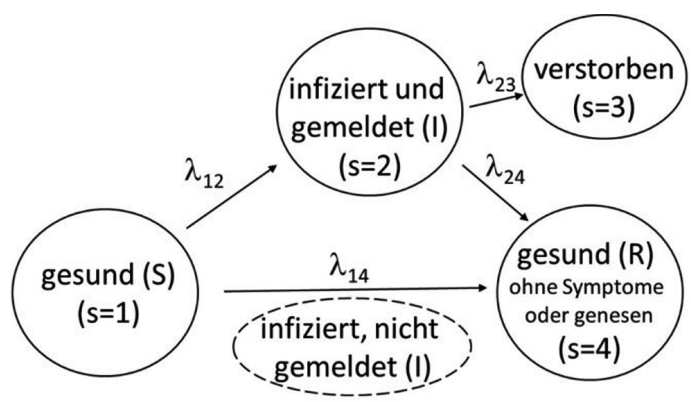

Abbildung 2: Das Modell aus Donsimoni et al. (2020a,b,c) mit deutschen Begriffen und Verbindung zum SIR-Modell Quelle: Eigene Darstellung

Das Wichtigste für eine Prognose sind die Übergangsraten $\lambda$, die in obiger Abbildung zwischen den Zuständen gezeigt sind. Die Rate $\lambda_{12}$ ist die Rate, mit der Individuen sich infizieren und Symptome entwickeln, so dass ein Test auf SARS-CoV-2 durchgeführt wird und positiv ausfällt. Mit einer Rate $\lambda_{14}$ infizieren sich Gesunde, entwickeln aber keine Symptome (oder gehen trotzdem nicht zum Arzt, oder die Symptome werden nicht als test-notwendig erachtet) und befinden sich dann im Zustand „genesen“. Mit der Rate $\lambda_{24}$ versterben infizierte (und gemeldete) Personen, mit der Rate $\lambda_{23}$ findet Genesung statt.

Die Infektionsrate $\lambda_{12}$ wird mit Daten des RKI geschätzt. Die Rate $\lambda_{14}$ folgt aus einer Annahme zum Anteil der stummen Infektionen (also Infektionen ohne Symptome, im Modell wäre dies der Wert 1-r). Während meist ein Anteil von 90 Prozent angenommen wird, wird in Robustheitsberechnungen auch der Effekt von Anteilen zwischen 80 und 99 Prozent untersucht. Die anderen Raten ergeben sich aus Beobachtungen zur Anzahl der Verstorbenen und der durchschnittlichen Genesungsdauer. 


\subsubsection{Vorgehen bei der Kalibrierung}

Die Schätzung erfolgt mithilfe statistischer Methoden, die in verschiedenen ökonometrischen Lehrbüchern oder in Artikeln beschrieben sind oder verwendet werden (beispielsweise Raue et al. 2013). Da die Datenmenge begrenzt ist und empirische Daten immer einen Fehler tragen, weisen auch die geschätzten Parameter eine Unsicherheit auf. Diese Unsicherheit wiederum setzt sich in die Unsicherheit der Modellvorhersagen fort (Kreutz 2012). Fragen der strukturellen Identifizierbarkeit von Parametern stellen sich ebenfalls (Raue 2009 sowie Launov und Wälde 2013, 2016). Das bedeutet für die üblichen SIR-Modelle, dass erst deutlich nach Verlassen der Phase des exponentiellen Wachstums verlässliche Vorhersagen möglich sind.

Donsimoni et al. (2020a, b) haben diesen Aspekt berücksichtigt, indem sie Annahmen darüber getroffen haben, wie hoch der Anteil der Infizierten an der Bevölkerung am Ende der Epidemie sein wird (im Modell der Parameter $\bar{\rho})$. Dazu können entweder historischen Analogien aus Tabelle 1 genommen oder Analogieschlüsse aus einfacheren SIR Modellen gezogen werden.

Beides ist höchst unzufriedenstellend. Es gibt zum einen keine historische Parallele zu Covid-19, zum anderen kein SIR-Modell, das für Covid-19 entwickelt worden ist. Aus diesem Grund haben die Autoren ein „optimistisches Hubei-Szenario" berechnet, in dem der Anteil $\bar{\rho}$ so hoch ist wie in der chinesischen Provinz Hubei aktuell (bei Annahme hoher Datenqualität und einem Ende der Epidemie in Hubei). Als Ergänzung haben sie ein „normales“ Szenario betrachtet, das weit höhere Werte für $\bar{\rho}$ annimmt. Für alle Szenarien wurde klar, dass die Epidemie im ungebremsten Verlauf (also ohne GPM) mindestens 4 bis 5 Monate dauert. Die Höhe der gleichzeitig Erkrankten schwankt je nach Szenario zwischen 200.000 und 1,2 Millionen Patienten. Damit war klar, dass ein ungebremster Verlauf das Gesundheitssystem sicher überlasten würde.

\section{Eine aktuelle Prognose und eine genauere Evaluation gesund- heitspolitischer Maßnahmen}

Wir können das obige Modell nun verwenden und über bisher vorliegende Analysen hinausgehend eine aktuelle Prognose mit Beobachtungen bis einschließlich 19. April vornehmen. Zum Verständnis der Idee sei darauf verwiesen, dass die Entwicklung von Covid-19 in Deutschland bisher drei regulatorische Phasen durchlief. Am Anfang kam es zu einer unkontrollierten Verbreitung der Infektio- nen und der damit einhergehenden Erkrankungen. Der Endpunkt dieser Phase kann auf den 16. März 2020 datiert werden. Ab diesem Zeitpunkt traten verschiedene „Kontaktsperren" in Kraft, unter anderem mit der Schließung der Schulen, der Absage großer Sportereignisse und vielem mehr. Diese Phase endete nach Ostern mit dem 20. April. Nach den Beschlüssen aus dem Treffen der Ministerpräsidenten der Länder und der Bundeskanzlerin vom 15. April ergaben sich verschiedene Lockerungen der GPM.

Wenn es uns gelingt, mithilfe statistischer und epidemiologischer Modelle eine Vorhersage zu treffen, wie die Entwicklung von Covid-19 in Deutschland verlaufen wäre, wenn es zum 20. April keine gesundheitspolitischen Änderungen gegeben hätte, dann wäre diese Vorhersage ein gutes Basisszenario. Letzteres könnte helfen, den Einfluss der Lockerungsmaßnahmen auf die Verbreitung von Covid-19 zu bestimmen. Gehen wir Schritt für Schritt vor.

\subsection{Referenzszenario und Prognosen auf der Ebene der Bundesrepublik}

\subsubsection{Schätzung eines Referenzszenarios}

Der Effekt von Lockerungen kann nur mithilfe eines Referenzszenarios verstanden werden, das den Effekt der Kontaktsperren beschreibt. Seit etwa einer Woche vor Ostern scheint der Verlauf der Covid-19 Infektionen in stabilen Bahnen zu verlaufen. Donsimoni et al (2020c) vermuteten in der Projektion vom 8. April bereits, dass ein Wendepunkt im Anstieg der Zahl der gemeldet Infizierten erreicht ist. Eine Aktualisierung vom 13. April (Wälde und Weiser 2020) wie auch Abbildung 7 unten (linker oberer Teil) bestätigten dies. Unsere aktuelle Prognose mit Daten bis 19. April für die Zahl der gemeldet Infizierten für die Bundesrepublik ist in Abbildung 3 dargestellt.

Diese Prognose zeigt das gesuchte Referenzszenario. Was wäre zu erwarten gewesen, wenn die Regeln am 20. April nicht geändert worden wären und wenn sich weiterhin alle Einwohner Deutschlands daran gehalten hätten? Wir würden den rot gestrichelten weiteren Verlauf beobachten. Wir werden in Kürze darauf zurückkommen. 


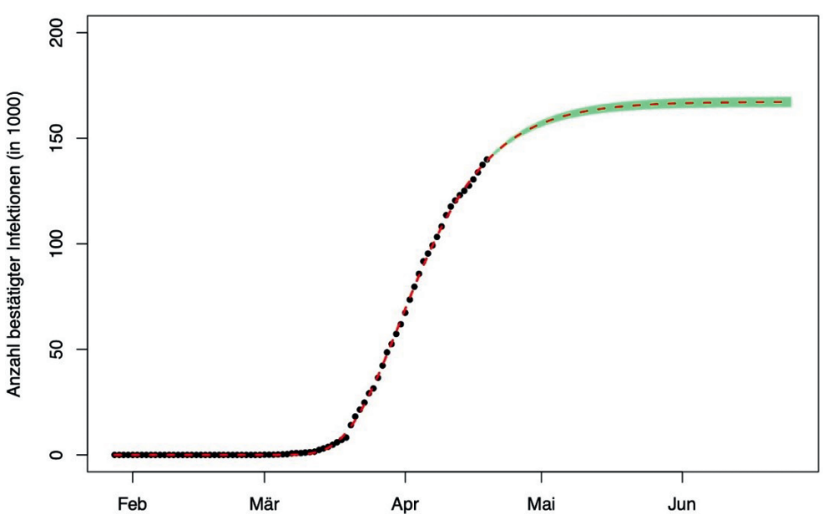

Abbildung 3: Die Zahl der gemeldet Infizierten (RKI) und der prognostizierte weitere Verlauf bei Beibehaltung der Kontaktregeln vor 20. April

Quelle: Eigene Darstellung

\subsubsection{Prognosen und Lockerungseffekte}

Die grundsätzliche Idee des Effekts einer Lockerung haben Donsimoni et al. (2020c) vorgestellt. Eine aktualisierte Version mit Kalibrierung unter Zuhilfenahme der Daten bis zum 19. April zeigt Abbildung 4.

Wir sehen, dass die Bestimmung der Modellparameter dazu führt, dass die Inzidenzen in der ersten regulatorischen Phase ohne jegliche öffentlichen Interventionen sehr gut durch die rote Kurve nachgezeichnet werden. Für die zweite Phase, in der GPM greifen, erfasst das Modell (gelbe Kurve) den durchschnittlichen Verlauf der täglich gemeldeten Neuerkrankungen gut. Im rechten Teil der Abbildung ist sichtbar, dass das Modell sowohl in der ersten als auch in der zweiten Phase die insgesamt je als Infizierte gemeldeten Personen sehr gut erklärt.
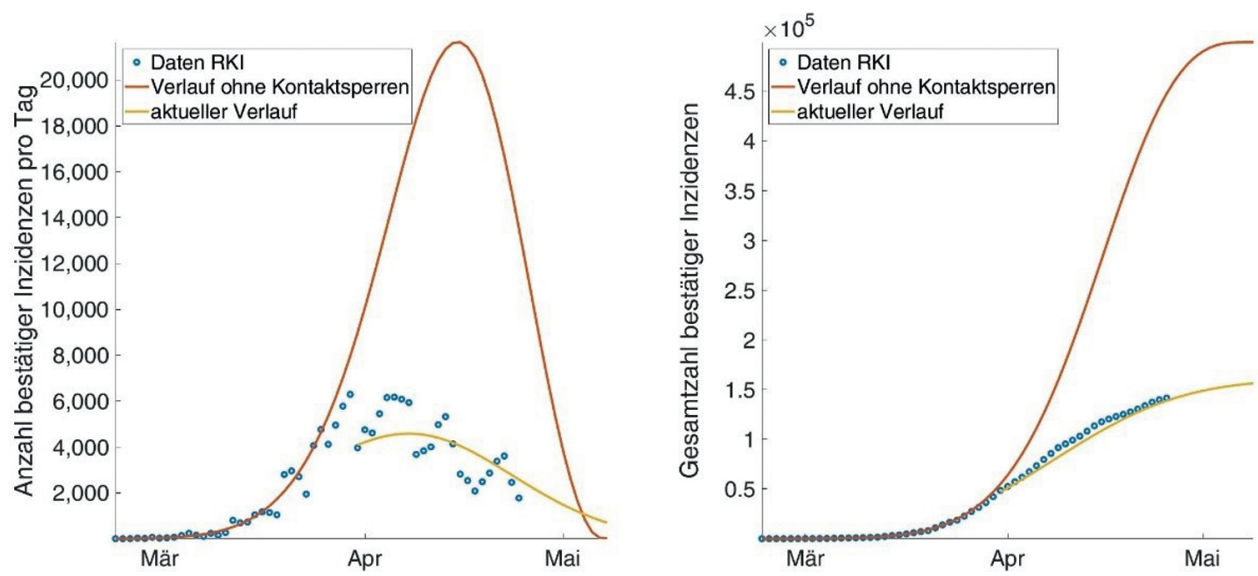

Abbildung 4: Anpassung des Modells an Covid-19-Daten in Deutschland. Anmerkung: Inzidenzen links und kumulierte Inzidenzen rechts Quelle: Eigene Darstellung

Quelle: Eigene Darstellung
Wir können dieses quantifizierte Modell nun verwenden, um verschiedene Szenarien zu betrachten. Diese erlauben uns $\mathrm{zu}$ verstehen, welche Verläufe Covid-19 in Deutschland hätte nehmen können und welche weiter möglich sind.

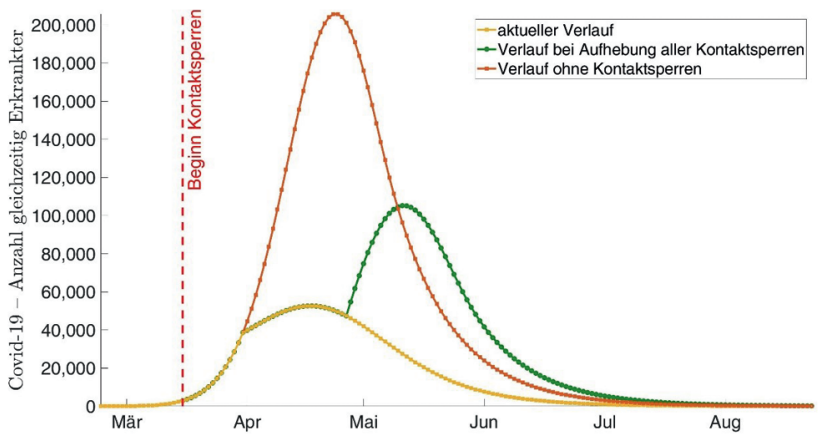

Abbildung 5: Verlauf von Covid-19 ohne jegliche Beschränkungen (rote Kurve), mit Kontaktsperren wie vom 16. März bis 19. April in Kraft (gelbe Kurve) und ein mögliches Szenario für die Zeit ab 27. April (grüne Kurve)

Abbildung 5 zeigt mit der roten Kurve die Prävalenz, also die Anzahl der gleichzeitig Erkrankten in Abwesenheit von GPM. In diesem „optimistischen Hubei-Szenario“ (Donsimoni et al. 2020 a, b) liegt die Prävalenz in der Spitze bei 200.000 Patienten. In weniger optimistischen Szenarien sind die Zahlen um einen Faktor 5 höher. Damit zeigt sich, dass ein ungebremster Verlauf von Covid-19 in Deutschland nie eine Option sein konnte. Der Effekt der GPM seit dem 16. März wird durch die gelbe Linie dargestellt. Es ergab sich eine erhebliche Abflachung der Kurve. Ein Vorteil in der Bestimmung der Parameter für den gelben Verlauf hier (und in Donsimoni et al. 2020c) lag im Vergleich zu Donsimoni et al. (2020 a,b) im Vorliegen von Daten für 
die Zeit während der Kontaktsperre. Somit ist der Modellverlauf in Abbildung 5 konsistent mit der Gompertzkurvenschätzung in Abbildung 3. Durch dieses Zusammenspiel von statistischen Methoden und theoretischen Modellen ist in Abbildung 5 also keine Annahmen mehr notwendig für den langfristen Anteil der Infizierten nach Ablauf der Epidemie (vgl. den weiter oben besprochenen Parameter $\bar{\rho}$ ).

Die grüne Linie zeigt einen möglichen Effekt der Lockerung der Kontaktsperren zum 20. April. Diese Effekte sind aufgrund der oben angesprochenen Verzögerung zwischen Erlass von GPM und gemeldeten Infektionen ab dem 27. April zu erwarten. Dies ist ein Beispiel für eine mögliche „zweite Welle“von Infektionen.

\subsection{Referenzszenarien und Evaluation gesundheitspolitischer Maßnahmen auf der Ebene der Bundesländer}

Kommen wir nun auf das Referenzszenario zur Evaluation gesundheitspolitischer Maßnahmen zurück. Wenn man die Effekte einer Lockerung verstehen möchte, ist ein solches Verständnis auf der Ebene der Bundesländer vermut-
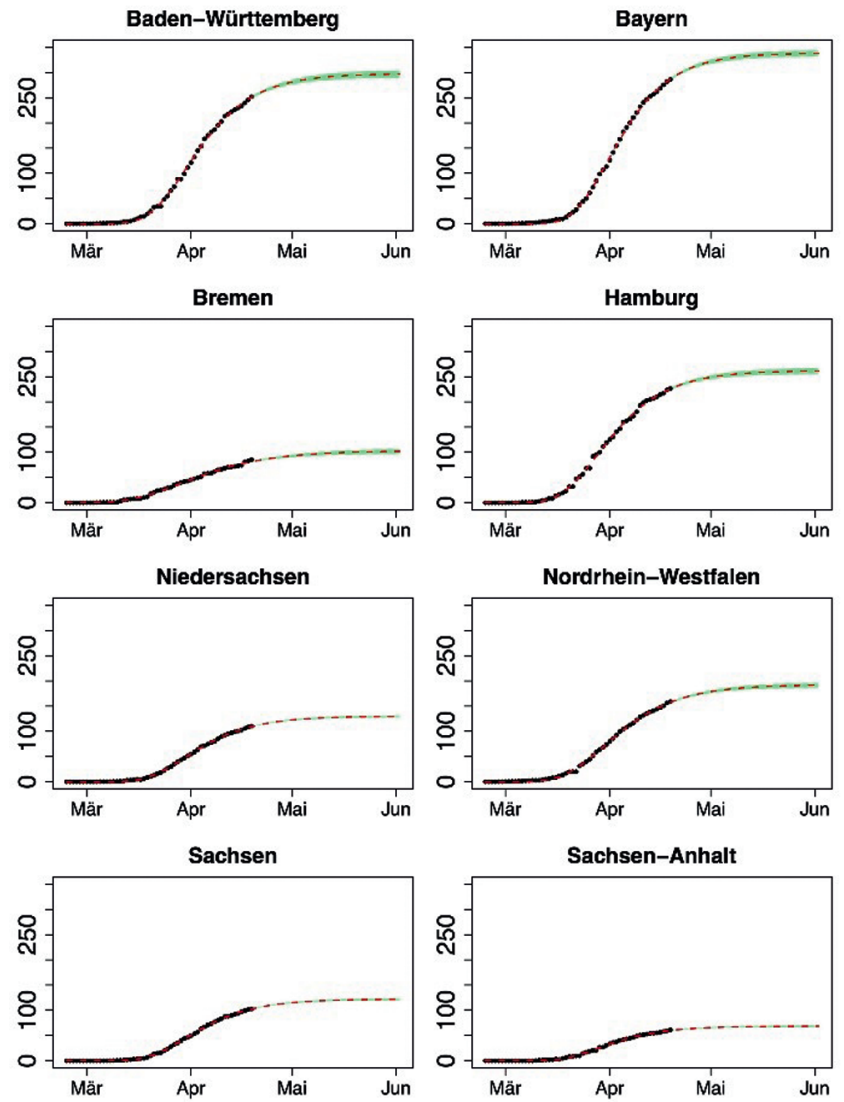

lich einfacher bzw. präziser. Deswegen stellen wir nun Referenzszenarien für 16 Bundesländer vor.

\subsubsection{Der bisherige Verlauf und eine hypothetische Prognose}

Abbildung 6 zeigt den Verlauf von gemeldeten CoV-2-Infektionen pro 100.000 Einwohner nach Bundesländern. $\mathrm{Zu}$ beachten ist bei dieser Abbildung, dass alle vertikalen Achsen gleich normiert sind. Somit ist ein schneller Vergleich der Infektionsanteile in den Bundesländern möglich.

Diese Abbildung zeigt den bisherigen Verlauf und die Prognose für den hypothetischen Fall der Beibehaltung der Kontaktregeln aus Phase 2 (16. März bis 19. April). Somit bekommen wir aus der Beobachtung des bisherigen Verlaufs ein Referenzszenario für die Bundesländer.

\subsubsection{Was sagen uns die Prognosen bezüglich gesundheitspolitischer Maßnahmen?}

Wir sehen nun endlich, wie Effekte einer Lockerung der Kontaktsperren verstanden werden können.
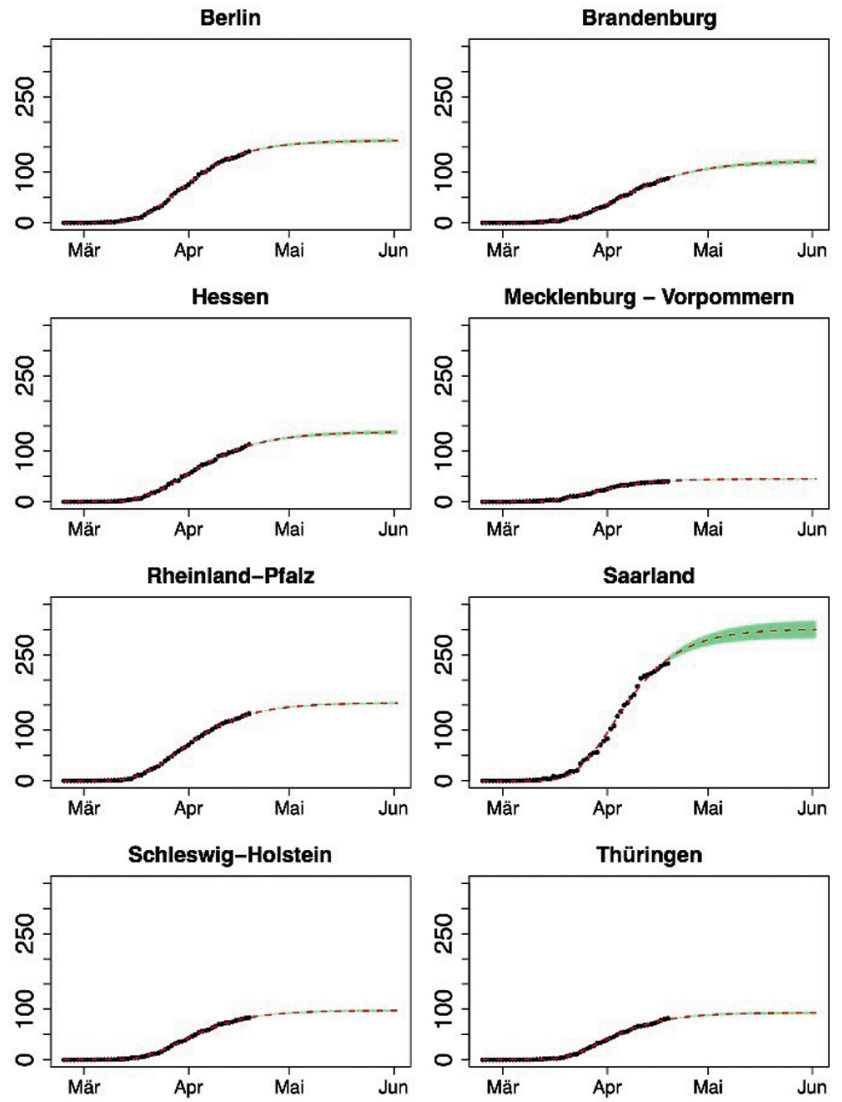

Abbildung 6: Gompertzkurven für alle Bundesländer Quelle: Eigene Darstellung 


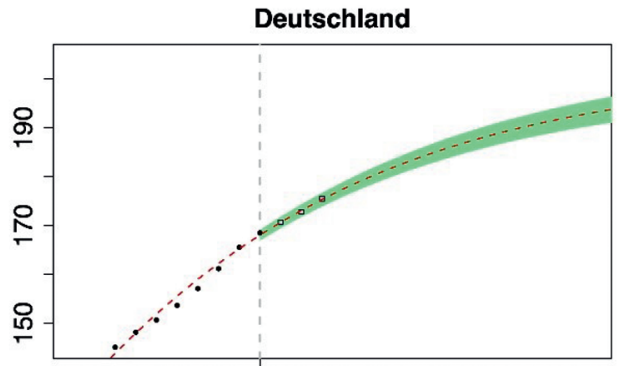

19. Apr

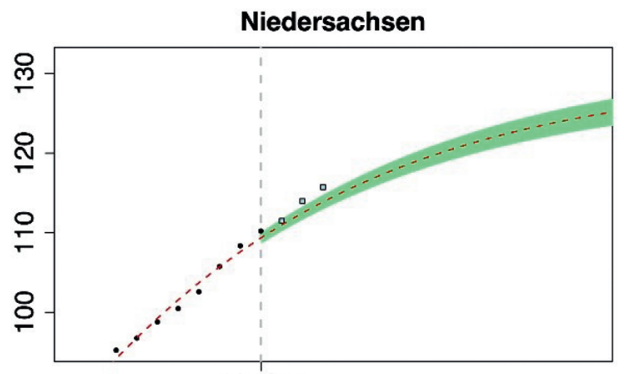

19. Apr

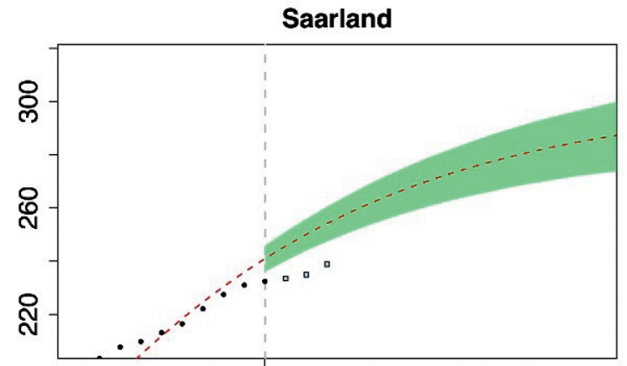

19. Apr

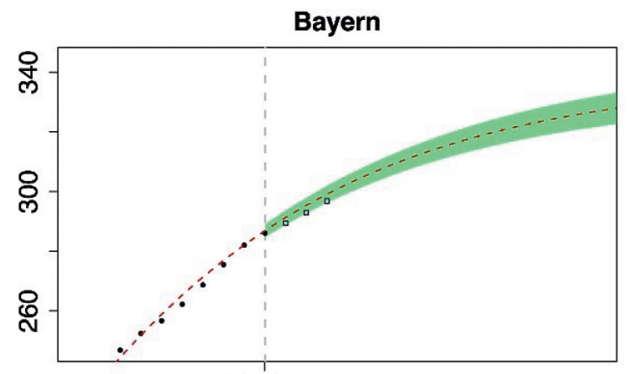

19. Apr

Abbildung 7: Für die Schätzung verwendete Beobachtungen (Punkte, RKI), Schätzung und Projektion (rote gestrichelte Linie), Konfidenzbereich (grüner Bereich) und neue Beobachtungen (Quadrate) für ausgewählte Bundesländer Quelle: Eigene Darstellung

Abbildung 7 zeigt Ausschnitte für die Bundesrepublik als Ganzes (links oben) und für ausgewählte Bundesländer. Zusätzlich zu den für das Basisszenario verwendeten Daten bis 19. April werden Beobachtungen vom 20. bis 22. April gezeigt. Wie wir sehen, liegen diese Beobachtungen für die Bundesrepublik im Konfidenzintervall der Vorhersage. Wir befinden uns in Deutschland insgesamt also immer noch in einem sehr stabilen Zustand, die neuen Regeln ab 20. April zeigen noch keine Wirkung auf die Verbreitung von Covid19. In einzelnen Bundesländern sehen wir jedoch Abweichungen nach oben und unten. Im Saarland verlangsamt sich die Ausbreitung, in Niedersachsen ist sie schneller als erwartet. In Bayern ist die Entwicklung stabil. Zu beachten ist aktuell allerdings noch, dass mit drei Beobachtungen relativ zum Basisszenario noch keine statistisch validen Aussagen im Sinne von „Lockerung der GPM war ein Erfolg“ oder „führt zu mehr Infektionen“ möglich ist. Ein Grund sind die üblichen Schwankungen über die Woche wegen beschränkter Meldemöglichkeiten am Wochenende. Es gilt also auf weitere Beobachtungen zu warten.

Wenn wir diese Idee mit anspruchsvolleren statistischen bzw. ökonometrischen Methoden in die Praxis umsetzen, wird es in Kürze möglich sein, die Effekte der gesundheitspolitischen Maßnahmen auf die Verbreitung von Covid-19 zu bestimmen. Dies sollte helfen, weitere Lockerungsmaßnahmen oder Korrekturen in Richtung strengere Regeln evidenzbasiert zu begründen. Die Aufgabe der Statistik in Zeiten von Covid-19 erscheint es also aktuell zu sein, effiziente von weniger effizienten (Lockerungen von) GPM zu unterscheiden. Analysen von Kosfeld et al. (2020) gehen in diese Richtung.

\section{Medizinische und ökonomische Kosten}

Anstelle einer Zusammenfassung sei hier erlaubt, die ökonomischen und medizinischen Kosten von Covid-19 zu betonen. Wie renommierte Mediziner betonen, starren wir auf Covid-19 „wie die Kaninchen auf die Schlange“. Auch wenn es nicht darum gehen kann, „Infizierte gegen Arbeitslose auszuspielen“, stellt sich natürlich die Frage, ob die öffentlichen und privaten Reaktionen angemessen oder übertrieben waren. Ein Problem liegt sicherlich in der täglichen Verfügbarkeit der Anzahl der gemeldet Infizierten. Dieses Vorliegen von Fakten stärkt die Aufmerksamkeit auf dieses Phänomen. Es liegen jedoch keine vergleichbaren täglichen Kenngrößen für wirtschaftliche Kosten oder auch die Kosten nicht stattgefundener anderer medizinischer Behandlungen vor.

Es ist eine Frage der Gewichtung einzelner Aspekte der Corona-Krise, wie die Antwort ausfällt. Wenn man alle ökonomischen und medizinischen Kosten in ausgefallene Arbeitstage oder qualitätskorrigierte Lebensjahre (QALYs) umrechnete, dann würden zumindest einige der Autoren 
dieses Artikels erwarten, dass die öffentlich getroffenen gesundheitspolitischen Maßnahmen ein bis zwei Jahre nach Ende der Pandemie bei Vorliegen aller notwendiger Daten einen positiven Saldo aufweisen. Aber auch dies ist eine Prognose.

Danksagung: Die Umstände der zurückliegenden Wochen führten zu einer Vielzahl von Gesprächen, die viele Fachgrenzen überbrückten. Die daraus gewonnenen Erkenntnisse haben uns sehr geholfen, diesen Text zu verfassen. Kommentare virtueller Workshop- und Vortragsteilnehmer flossen ebenfalls ein. Ein besonderer Dank geht an Silke Bernardi für die Unterstützung nicht nur bei der Erstellung dieses Artikels. Gefördert durch die Deutsche Forschungsgemeinschaft (DFG) im Rahmen der Exzellenzstrategie des Bundes und der Länder EXC-2189 - Projekt ID: 390939984.

\section{Literaturverzeichnis}

Adam, D. (2020), Special report: The simulations driving the world's response to COVID-19, How epidemiologists rushed to model the coronavirus pandemic, Nature 580, S. 316-18.

Alfani, G. (2020), Pandemics and asymmetric shocks: Lessons from the history of plagues, VoxEU.org.

Alfani, G. und T. Murphy (2017), Plague and lethal epidemics in the preindustrial world, Journal of Economic History 77(1), S. 314-43.

Allen, L. J. S. (2008), An introduction to stochastic epidemic models, in F. Brauer und P. van den Driessche (Hrsg.), Jianhong Wu Mathematical Epidemiology, Berlin, Heidelberg, Springer, S. 81-130.

Bai, J. (1997), Estimation of a change point in multiple regression models, The Review of Economics and Statistics 79(4), S. 551-63.

Bai, J. und P. Perron (1998), Estimating and testing linear models with multiple structural changes, Econometrica 66(1), S. 47-78.

Boeri, T., A. Caiumi und M. Paccagnella (2020), Mitigating the worksecurity trade-off while rebooting the economy, VoxEU.org.

Center for Disease Control (2020), Symptoms of coronavirus, online verfügbar unter https://www.cdc.gov/coronavirus/2019-ncov/ symptoms-testing/symptoms.html.

Clayville, L. R. (2011), Influenza update: A review of currently available vaccines, Pharmacy and Therapeutics 36(10), S. 659-84.

Diamond, P. A. (1982), Aggregate demand management in search equilibrium, Journal of Political Economy 90, S. 881-94.

Donsimoni, J. R., R. Glawion, B. Plachter und K. Wälde (2020a), Projecting the spread of COVID-19 for Germany, German Economic Review, im Erscheinen.

Donsimoni, J. R., R. Glawion, B. Plachter und K. Wälde (2020b), Projektion der COVID19-Epidemie in Deutschland, Wirtschaftsdienst 100, S. $272-76$.

Donsimoni, J. R., R. Glawion, B. Plachter, C. Weiser und K. Wälde (2020c), Should contact bans be lifted in Germany? A quantitative prediction of its effects, online verfügbar unter https:// www.medrxiv.org/content/10.1101/2020.04.10.20060301v1.

Ferguson, N. M. et al. (2020), Impact of non-pharmaceutical interventions (NPIs) to reduce COVID-19 mortality and healthcare demand, Imperial College COVID-19 Response Team.
Fragaszy, E. B. et al. (2017), Cohort profile: The Flu Watch Study, International Journal of Epidemiology 46(2), S. 1-11.

Fraser, C., D. A. T. Cummings, D. Klinkenberg, D. S. Burke und N. M. Ferguson (2011), Influenza Transmission in Households During the 1918 Pandemic, American Journal of Epidemiology 174(5), S. 505-14.

Glezen, W.P. (1996), Emerging infections: Pandemic influenza, Epidemiologic Reviews, 18(1), S. 64-76.

Hartl, T., K. Wälde und E. Weber (2020a). Measuring the impact of the German public shutdown on the spread of COVID-19, Covid Economics: Vetted and Real-Time Papers 1(1), S. 25-32.

Hartl, T., K. Wälde und E. Weber (2020b). Measuring the impact of the German public shutdown on the spread of COVID-19, Voxeu. org.

Hethcote, H. (2000), The Mathematics of infectious diseases, SIAM Review 42(4), S. 559-653.

Jackson, C. (2009), History lessons: The Asian Flu pandemic, British Journal of General Practice 59(265), S. 622-23.

Johns Hopkins University (2020), COVID-19 Dashboard, Center for Systems Science and Engineering, online verfügbar unter https://coronavirus.jhu.edu/map.html.

Juliano, A. D. et al. (2018), Estimates of 3 global seasonal influenzaassociated respiratory mortality: a modelling study, The Lancet 391, S. 1285-300.

Kermack, W. O. und A. G. McKendrick (1927), A contribution to the mathematical theory of epidemics, Proceedings of the Royal Society 115(772), S. 700-21.

Kilbourne, E. D. (2006), Influenza pandemics of the 20th century, Emerging Infectious Diseases 12(1), S. 9-14.

Kosfeld, R., T. Mitze, J. Rode und K. Wälde (2020), The infection effects of public health measures, in Arbeit.

Kreutz, C., A. Raue und J. Timmer (2012), Likelihood based observability analysis and confidence intervals for predictions of dynamic network models, BMC Systems Biology 6(120).

Lauer, S. A. et al. (2020), The incubation period of Coronavirus disease 2019 (COVID-19) from publicly reported confirmed cases: Estimation and application, Annals of Internal Medicine.

Launov, A. und K. Wälde (2013), Estimating incentive and welfare effects of non-stationary unemployment benefits, International Economic Review 54, S. 1159-98.

Launov, A. und K. Wälde (2016), The employment effect of reforming a public employment agency, European Economic Review 84, S. $140-64$.

Linton, N. M. et al. (2020), Incubation period and other epidemiological characteristics of 2019 novel Coronavirus infections with right truncation: A statistical analysis of publicly available case data, Journal of Clinical Medicine 9(2), 538.

Merkl, C. und E. Weber (2020), Rescuing the labour market in times of COVID-19: Don't forget new hires!, VoxEU.org.

Mortensen, D. T. (1982), Property rights and efficiency in mating, racing, and related games, American Economic Review 72, S. $968-79$.

Pissarides, C. A. (1985), Short-run equilibrium dynamics of unemployment vacancies, and real wages, American Economic Review 75, S. $676-90$.

Raue, A. et al. (2009), Structural and practical identifiability analysis of partially observed dynamical models by exploiting the profile likelihood, Bioinformatics 25, S. 1923-29.

Raue, A. et al. (2013), Lessons learned from quantitative dynamical modeling in systems biology, PLoS ONE 8(9), e74335. 
Robert-Koch-Institut (RKI) (2019), Bericht zur Epidemiologie der Influenza in Deutschland Saison 2018/19, online verfügbar unter https://influenza.rki.de/Saisonberichte/2018.pdf.

Saunders-Hastings, P. R. und D. Krewski (2016), Reviewing the History of Pandemic Influenza: Understanding Patterns of Emergence and Transmission, Pathogens 5(4), S. 1-19.

Scholz, S., O. Damm, U. Schneider, B. Ultsch, O. Wichmann und W. Greiner (2019), Epidemiology and cost of seasonal influenza in Germany - a claims data analysis, BMC Public Health 19 (1090).

Starling, A. (2006), Plague, SARS, and the Story of Medicine in Hong Kong. Hongkong, HK University Press.

Taubenberger, J. K. (2006), The origin and virulence of the 1918 "Spanish" influenza virus, Proceedings of the American Philosophical Society 150(1), S. 86-112.

Taubenberger, J. K. und D. M. Morens (2006),1918 Influenza: The mother of all pandemics, Emerging Infectious Diseases 12(1), S. $15-22$.

Wälde, K. (2020), Corona-Blog, Einschätzung vom Freitag, 20. März, online verfügbar unter https://www.macro.economics.unimainz.de/2020/03/20/einschatzung-vom-freitag-20-marz/.
Wälde, K. und Weiser, C. (2020), Weiterhin rückläufige Zuwachsraten - Einschätzung vom 13. April, online verfügbar unter https://www.macro.economics.uni-mainz.de/2020/04/13/ weiterhin-rucklaufige-zuwachsraten-einschatzung-vom-13april/.

Wilder-Smith, A., C. Chiew und V. Lee (2020), Can we contain the COVID-19 outbreak with the same measures as for SARS?, The Lancet. Infectious Diseases, online verfügbar unter https://www. ncbi.nlm.nih.gov/pmc/articles/PMC7102636/.

World Health Organization (2004), Summary of probable SARS cases with onset of illness from 1 November 2002 to 31 July 2003, online verfügbar unter https://www.who.int/csr/sars/country/ table2004_04_21/en/.

Zhang, S., M. Diao, W. Yu, L. Pei, Z. Lin und D. Chen (2020), Estimation of the reproductive number of novel coronavirus (COVID-19) and the probable outbreak size on the Diamond Princess cruise ship: A data-driven analysis, International Journal of Infectious Diseases 93, S. 201-04. 